\title{
PERMUTATIONAL PRODUCTS OF LATTICE ORDERED GROUPS ${ }^{1}$
}

\author{
N. R. REILLY \\ (Received 15 April 1969; revised 10 September 1969) \\ Communicated by B. Mond
}

Let $H$ be a group, let $\left\{G_{i}: i \in I\right\}$ be a set of groups and, for each $i$, let $\theta_{i}$ be a a monomorphism: $H \rightarrow G_{i}$, with $H \theta_{i}=H_{i}$. We call such a system of groups and monomorphisms an amalgam and dencte it by $\left[G_{i} ; H ; \theta_{i} ; H_{i}\right]$. By an embedding of the amalgam into a group $G$ is meant a set of monomorphisms $\varphi_{i}: G_{i} \rightarrow G$ such that $\theta_{i} \varphi_{i}=\theta_{j} \varphi_{j}$, for all $i, j$ and $G_{i} \varphi_{i} \cap G_{j} \varphi_{j}=H \theta_{k} \varphi_{k}$, for all $i, j, k$.

It is known (B. H. Neumann [9]) that the amalgam $\left[G_{i} ; H ; \theta_{i} ; H_{i}\right]$ can be embedded in a group. It is also known (J. M. Howie [7]) that if the $G_{i}$ are just semigroups and the $H_{i}$ are almost unitary subsemigroups then the amalgam may be embedded in a semigroup.

If $G$ is an 1-group (lattice ordered group), a subgroup which is also a sublattice is called an 1-subgroup. If $H$ is an 1-subgroup of $G$ and if the mapping $C \rightarrow C \cap H$ is a one-to-one correspondence between the lattice of 1-subgroups of $G$ and those of $H$ then Conrad [2] called $G$ an a-extension of $H$. In his discussion of a-extensions Conrad has shown that amalgams of the form $\left[G_{i} ; H ; \theta_{i} ; H_{i}\right]$ are embeddable where the $G_{i}$ are $a$-extensions of the $H_{i}$ and the $H_{i}$ all belong to one of a number of classes of 1-groups. In a sense, the second section of this note considers the other extreme to Conrad's results. In section two, we consider the problem of embedding the amalgam $\left[G_{i} ; H ; \theta_{i} ; H_{i}\right]$, where $H$ and $G_{i}$ are 1-groups, the $H_{i}$ are convex 1-subgroups and the $\theta_{i}$ are 1-monomorphisms (lattice and group monomorphisms), into an 1-group $G$. It is shown that if, for each $i, H_{i}$ is in the centre of $G_{i}$ then the amalgam is embeddable and if each $G_{i}$ is abelian then the amalgam may be embedded in an abelian 1-group.

The approach combines the permutational product of groups with the representation of an 1-group as an 1-subgroup of the group of automorphisms of an ordered set due to Holland [6]. If the $G_{i}$ are 0-groups (totally ordered groups) and the $H_{i}$ are normal then the standard permutational product may be used. In general this does not yield an C-group although it will if the $G_{i}$ are abelian.

In more general situations (e.g. [10]), consideration has been given to a weaker amalgamation embedding. Let us say that an amalgam $\left[G_{i} ; H ; \theta_{i} ; H_{i}\right]$, where the

1 This research was supported in part by N.R.C. Grant No. A-4044. 
$G_{i}$ are 1-groups and the $H_{i}$ are convex 1-subgrcups, is weakly embeddable if there exists an 1-group $G$ and 1-monomorphisms $\theta_{i}: G \rightarrow G_{i}$ such that $\theta_{i} \varphi_{i}=\theta_{j} \varphi_{j}$, for all $i, j$ : that is, we drop the intersection preserving requirements.

K. Pierce (University of Wisconsin) has provided an example (unpublished) to show that the amalgam $\left[G_{i} ; H ; \theta_{i} ; H_{i}\right]$, where the $H_{i}$ are abelian 1 -subgroups of the 1-groups $G_{i}$, cannot, in general, be even weakly embedded in an 1-group.

Unfortunately, on account of the restrictions placed on the subgroups $H_{i}$ to be amalgamated, these results do not permit the kind of interesting applications treated by B. H. Neumann in (9) and by Higman, Neumann and Neumann in [5].

Finally, I would like to thank R. Gregorac for his many comments and criticisms, especially for pointing out an over ambitious claim in Theorem 4.1 as originally stated.

\section{Preliminary results}

We refer the reader to [1] and [4] for basic results on 1-groups.

For any totally ordered set $X$, we denote by $P(X)$ the lattice ordered group of automorphisms (that is, order preserving permutatio us) of $X$.

A convex 1-subgroup $A$ of an 1-group $G$ is called prime if the set $R(A)$ of right ccsets of $A$ can be totally ordered by defining $A+x \leqq A+y$ if $a+x \leqq y$ for some $a \in A$. For further discussion of prime subgroups see [3] and [6]. Whenever $A$ is a prime subgroup of an 1 -group $G, G / A$ will denote the set cf right cosets of $A$ ordered in this way. (Of course, in general a prime subgroup is not normal.) The mapping $\rho: g \rightarrow \rho_{g}$ of $G$ into $P(G / A)$ defined by

$$
(A+x) \rho_{p}=A+x+g
$$

is an 1-homomorphism. We call $\rho$ the Holland representation of $G$ on $G / A$.

Prime subgroups are plentiful. If $g \in G$ and $G_{\gamma}$ is a maximal convex 1-subgroup with respect to not containing $g$ then $G_{\gamma}$ is a prime subgroup. Denote by $\sum_{i \in I} \boxplus A_{i}$ the unrestricted cardinal sum of 1-groups $A_{i}$. If $\left\{G_{\gamma}: \gamma \in \Gamma(G)\right\}$ is the set of prime subgroups of $G$ and $\theta: G \rightarrow \sum_{\gamma \in \Gamma(G)} \oplus P\left(G / G_{\gamma}\right)$ is the product of the Holland representations on the $G / G_{\gamma}$ then $\theta$ is an 1-isomorphism. (For details of this representation see Holland [6]).

If $\left\{G_{\gamma}: \gamma \in \Gamma(G)\right\}$ is the set of all prime subgroups of $G$, if we totally order $\Gamma(G)$ and then order the set of all left cosets $C(G)$ of all prime subgroups of $G$ by $G_{\gamma}+a>G_{\gamma}^{\prime}+a^{\prime}$ if $\gamma>\gamma^{\prime}$ or $\gamma=\gamma^{\prime}$ and $G_{\gamma}+a>G_{\gamma}+a^{\prime}$ (in the order defined in the previous paragraph) then we can obtain a faithful representation of $G$ as an 1-subgroup of $P(C(G)$ ), (Holland [6]).

Lemma 1.1 (Lloyd [8]). Let $A \subseteq B$ be prime subgroups of a 1-group $G$. Then there exists an order isomorphism $\eta^{\prime}$ of $G / A$ onto $B / A \times G / B$, where $B / A \times G / B$ is ordered lexicographically from the right. This induces an 1-isomorphism $\eta$ of $P(G / A)$ onto $P(B / A \times G / B)$. 
The isomorphism $\eta^{\prime}$ in Lemma 1.1 is defined as follows. Let $\left\{b_{i}: i \in I\right\}$ be a set of representatives of the right cosets of $B$ in $G$. Let $A+x \in G / A$ and $B+x=$ $B+b_{i}$. Then $(A+x) \eta^{\prime}=\left(A+x-b_{i}, B+b_{i}\right)$. Of course, if $A=B$ then $B / A \times G / B$ is trivially isomorphic to $G / B=G / A$.

An 1-ideal of an 1-group is a normal convex 1-subgroup. The following lemma is routine.

Lemma 1.2. Let $G$ be an 1-group, $A$ an 1-ideal and $B$ a prime subgroup of $G$. Then there exists an order isomorphism $\zeta^{\prime}$ of $(B+A) / B$ onto $A / A \cap B$.

Proof. We take $\zeta^{\prime}$ to be the obvicus isomorphism defined by $\left(B+x^{\prime}\right) \zeta^{\prime}=$ $(A \cap B)+x$, where $x \in A$ and $B+x^{\prime}=B+x$.

Let $G$ be an 1-group, $H$ an 1-ideal and $G_{\gamma}$ a prime subgroup of $G$. Let $\xi_{\gamma}$ be the Holland representation of $G$ on $G / G_{\gamma}$. Let $\eta_{\gamma}$ be the 1-isomorphism of $P\left(G / G_{\gamma}\right)$ onto $P\left(\left(G_{\gamma}+H\right) / G_{\gamma} \times G /\left(G_{\gamma}+H\right)\right)$ defined as in Lemma 1.1. Let $\zeta_{\gamma}$ be the 1-isomorphism of $P\left(\left(G_{\gamma}+H\right) / G_{\gamma} \times G /\left(G_{\gamma}+H\right)\right)$ onto $P\left(H / H \cap G_{\gamma} \times G /\left(G_{\gamma}+H\right)\right)$ induced by the order isomorphism of $\left(G_{\gamma}+H\right) / G_{y}$ onto $H / H \cap G_{\gamma}$ defined as in Lemma 1.2. Thus $\xi_{\gamma} \eta_{\gamma} \zeta_{\gamma}$ is an 1-homomorphism of $G$ into $P\left(H / H \cap G_{\gamma} \times G /\left(G_{\gamma}+H\right)\right)$. We write $\Psi_{\gamma}=\xi_{\gamma} \eta_{\gamma} \zeta_{\gamma}$.

\section{Permutational products}

Let $\left\{G_{i} \quad i \in I\right\}$ be a set of distinct 1-groups and, for each $i$, let $H_{i}$ be a convex 1-subgroup of $G_{i}$ contained in the centre of $G_{i}$. Let $H$ be an 1-group and, for each $i, \theta_{i}: H \rightarrow H_{i}$ be an 1 -isomorphism. We wish to embed the amalgam $\left[G_{i} ; H ; \theta_{i} ; H_{i}\right]$ in an 1-group. For any prime subgroup $H_{\gamma}$ of $H, H_{\gamma} \theta_{i}$ is a prime subgroup of $H_{i}$ and $\theta_{i}$ induces an order isomorphism of $H / H_{\gamma}$ onto $H_{i} / H_{\gamma} \theta_{i}$, which we also denote by $\theta_{i}$. We introduce a dummy symbol 1 .

We denote by $\left\{H_{\gamma}: \gamma \in \Gamma(H)\right\}\left(\left\{H_{i, \gamma_{i}}: \gamma_{i} \in \Gamma\left(H_{i}\right)\right\}\right)$ the set of prime subgroups of $H\left(H_{i}\right)$. Let $\Gamma(H)$ be endowed with some total order, then we can order the set of right cosets $C(H)$ of the prime subgroups of $H$ by defining $H_{\gamma}+x>H_{\gamma}^{\prime}+x^{\prime}$ if either $\gamma>\gamma^{\prime}$ or $\gamma=\gamma^{\prime}$ and $H_{\gamma}+x>H_{\gamma}+x^{\prime}$. We extend the order on $C(H)$ to $C(H)^{1}=C(H) \cup\{1\}$ by defining 1 to be the least element.

Similarly, let $C\left(H_{i}\right)$ denote the set of right cosets of the prime subgroups of $H_{i}$. Then $\theta_{i}$ determines a bijection of $C(H)$ onto $C\left(H_{i}\right)$ which is an order isomorphism when restricted to the cosets of an individual prime subgroup of $H$. Thus $\theta_{i}$ induces an ordering of $C\left(H_{i}\right)$ extending that on the cosets of the individual prime subgroups.

For each $i \in I$, let $\left\{G_{i, \gamma}: \gamma \in \Gamma\left(G_{i}\right)\right\}$ denote the set of prime subgroups of $G_{i}$ and let $\left\{G_{i, \gamma}: \gamma \in \Gamma_{1}\left(G_{i}\right)\right\}$ denote the set of prime subgroups of $G_{i}$ containing $H_{i}$. Order $\Gamma_{1}\left(G_{i}\right)$ and then the set of cosets $C\left(G_{i}\right)$ of the prime subgroups $G_{i, \gamma}$ with $\gamma \in \Gamma_{1}\left(G_{i}\right)$ just as $C(H)$ was ordered.

It has been shown by Conrad [3] that, for any convex 1-subgroup $K$ of an 
1-group $L$, the mapping $\sigma: A \rightarrow A \cap K$ is a bijection from the set of prime subgroups of $L$ that do not contain $K$ to those of $K$. So, for each $i$, we denote by $\sigma_{i}$ the correspondence between the prime subgroups of $G_{i}$ not containing $H_{i}$ and the prime subgroups of $H_{i}$.

We define a subset $T$ of the cartesian product of $C(H)^{1}$ and the sets $C\left(G_{i}\right)$, considered as the set of (choice) functions $p$ from $J=I \cup\{\alpha\}$ such that $p(\alpha) \in C(H)^{1}$ and $p(i) \in C\left(G_{i}\right), i \in I$. A function $p$ belongs to $T$ if

$\begin{array}{ll}\text { either } & p(\alpha)=1 \\ \text { or } & p(\alpha)=H_{\gamma}+h, p(i)=G_{i, \gamma}+g_{i} \\ \text { and } & H_{\gamma} \theta_{i} \sigma_{i}^{-1}+H_{i}=G_{i, \gamma}\end{array}$

Note: By the definition of $C\left(G_{i}\right)$, the only prime subgroups of $G_{i}$ whose cosets appear as $p(i)$, for some $p$, are the prime subgroups of $G_{i}$ containing $H_{i}$.

We choose a fixed set of representatives $g_{i \gamma}, g_{i \gamma}^{\prime}, \cdots$ of the cosets in $G_{i}$ of the prime subgroups $G_{i, \gamma}$ of $G_{i}$, as required for the application of Lemma 1.1.

Totally order $T$ as follows: first well order $I$ : then define $p>q$ if and only if either $p(i)>q(i)$ for the first element $i \in I$ for which $p(i) \neq q(i)$ or $p(i)=q(i)$, for all $i \in I$, and $p(\alpha)>q(\alpha)$.

We now represent each $G_{i}$ as a 1-subgroup of $P(T)$.

For each $i \in I$ and each $\gamma \in \Gamma(H)$ let $\psi_{i \gamma}$ and $\xi_{i \gamma}$ be the $l$-homomorphisms of $G_{i}$ into $P\left(H_{i} / H_{\gamma} \theta_{i} \times G_{i} / H_{\gamma} \theta_{i} \sigma_{i}^{-1}+H_{i}\right)$ and $P\left(G_{i} / H_{\gamma} \theta_{i} \sigma_{i}^{-1}+H_{i}\right)$, respectively, defined by analogy with the definition of $\psi_{\gamma}$ and $\xi_{\gamma}$ in Section 1 . Then we define a mapping $\phi_{i}: G_{i} \rightarrow P(T)$ as follows: let $p \in T$.

If $p(x)=1$, then define $p\left(g \phi_{i}\right)=q$ where

$$
q(\alpha)=1=p(\alpha), q(i)=G_{i, \gamma}+g_{i \gamma}+g, q(j)=p(j) \text { for } j \neq i .
$$

If $p(\alpha) \neq 1$ and $g \in G_{i}$, suppose that

$$
\left(p(\alpha) \theta_{i}, G_{i, \gamma}+g_{i \gamma}\right) g \psi_{i \gamma}=\left(y \theta_{i}, G_{i, \gamma}+g_{i \gamma}^{\prime}\right) .
$$

Then define $p\left(g \phi_{i}\right)=q$ where

$$
q(\alpha)=y, q(i)=G_{i, \gamma}+g_{i \gamma}^{\prime}, q(j)=p(j) \text { for } j \neq i .
$$

It is not difficult to see that $\phi_{i}$ is faithful and is essentially just a product of Holland representations and so is an 1-isomorphism.

Now consider $h \theta_{i} \phi_{i}, h \theta_{j} \phi_{j}$ for $h \in H, i, j \in I$. Let $p \in T$.

Case (i), $p(\alpha)=1$. Then $p\left(h \theta_{i} \phi_{i}\right)=q$, where

$$
\begin{aligned}
& q(\alpha)=p(\alpha), q(i)=G_{i, \gamma}+g_{i \gamma}+h \theta_{i}=G_{i, \gamma}+g_{i \gamma}=p(i), \\
& q(r)=p(r), \text { for } r \neq i,
\end{aligned}
$$

since $h \theta_{i}$ is in the centre of $G_{i}$ and $H_{i} \subseteq G_{i, \gamma}$. Thus $p\left(h \theta_{i} \phi_{i}\right)=p$ and, similarly, $p\left(h \theta_{j} \phi_{j}\right)=p$. 
Case (ii), $p(\alpha)=H_{\gamma}+a$. Then $p\left(h \theta_{i} \phi_{i}\right)=q$, say, where $q(r)=p(r)$ for $r \neq \alpha, i$. Now $q(i)$ and $q(\alpha)$ are determined as in Lemmas 1.1, 1.2, by

$$
\left(H_{\gamma} \theta_{i}+a \theta_{i}, G_{i, \gamma}+g_{i \gamma}\right)\left(h \theta_{i} \psi_{i \gamma}\right)
$$

and this is determined by

$$
\left(H_{\gamma} \theta_{i} \sigma_{i}^{-1}+a \theta_{i}+g_{i \gamma}\right)\left(h \theta_{i}\right) \xi_{i \gamma}
$$

Now,

$$
\begin{aligned}
\left(H_{\gamma} \theta_{i} \sigma_{i}^{-1}+a \theta_{i}+g_{i \gamma}\right)\left(h \theta_{i}\right) \xi_{i \gamma} & =H_{\gamma} \theta_{i} \sigma_{i}^{-1}+a \theta_{i}+g_{i \gamma}+h \theta_{i} \\
& =H_{\gamma} \theta_{i} \sigma_{i}^{-1}+(a+h) \theta_{i}+g_{i \gamma}
\end{aligned}
$$

since $h \theta_{i}$ is in the centre of $G_{i}$. Hence,

Thus

$$
\left(H_{\gamma} \theta_{i}+a \theta_{i}, G_{i, \gamma}+g_{i \gamma}\right) h \theta_{i} \psi_{i \gamma}=\left(H_{\gamma} \theta_{i}+(a+h) \theta_{i}, G_{i, \gamma}+g_{i \gamma}\right) \text {. }
$$

$$
q(\alpha)=H_{\gamma}+a+h \text { and } q(r)=p(r), \text { for } r \neq \alpha
$$

Similarly, we will find that $p\left(h \theta_{j} \varphi_{j}\right)=q$.

Thus $\theta_{i} \varphi_{i}=\theta_{j} \varphi_{j}$ for all $i, j$ while clearly, for any $i, j, k$,

$$
G_{i} \varphi_{i} \cap G_{j} \varphi_{j}=\bigcap_{i} G_{i} \varphi_{i}=H \theta_{k} \varphi_{k}
$$

We call the 1-subgroup of $P(T)$ generated by the 1 -subgroups $G_{i} \varphi_{i}$ the 1-permutational product of the 1-groups $G_{i}$ with the 1-subgroups $H_{i}$ amalgamated. Thus we have shown

THEOREM 2.1. Let $\left\{G_{i}: i \in I\right\}$ be a set of 1-groups, $\left\{H_{i}: i \in I\right\}$ a set of convex 1-subgroups such that $H_{i}$ is in the centre of $G_{i}$, let $H$ be an 1-group and, for each $i$, $\theta_{i}$ be an 1-isomorphism of $H$ onto $H_{i}$. Then there exists an 1-group $P$, the 1-permutational product of the 1-groups $G_{i}$ with the 1-subgroups $H_{i}$ amalgamated, and 1 isomorphisms $\varphi_{i}$ of $G_{i}$ into $P$ such that $\theta_{i} \varphi_{i}=\theta_{j} \varphi_{j}$ for all $i, j, G_{i} \varphi_{i} \cap G_{j} \varphi_{j}=$ $\bigcap_{i} G_{i} \varphi_{i}=H \theta_{k}$, for all $i, j, k$, and $P$ is generated as an 1-group by $\left\{G_{i} \varphi_{i}\right\}$. Moreover, if all the $G_{i}$ are abelian, then so is $P$.

The last remark of the theorem follows since it can easily be shown that the elements of $G_{i} \varphi_{i}$ commute with the elements of $G_{j} \varphi_{j}$ (provided $i \neq j$ ). Thus, if the $G_{i}$ are all abelian then $\left\{G_{i} \varphi_{i}\right\}$ generates an abelian subgroup of $P$ and hence an abelian 1-subgroup of $P$.

Note: We point out that the class of 1-groups with non-trivial convex central 1 -subgroups does contain non-abelian members. Suppose that $G$ is a torsion free nilpotent group with lower central series

$$
G=\gamma_{1}(G) \supset \gamma_{2}(G) \supset \cdots \supset \gamma_{n+1}(G)=1
$$

and torsion free factors $\gamma_{i}(G) / \gamma_{i+1}(G)$. Let the torsion free abelian group $\gamma_{n}(G)$ be endowed with some lattice order: provided the rank of $\gamma_{n}(G)$ is not one, one could 
ensure that this is not a total order. Let each torsion free abelian factor $\gamma_{i}(G) /$ $\gamma_{i+1}(G), i \neq n$, be endowed with some total order. Then the order defined on $G$ as follows is a lattice order, with respect to which $G$ is an 1-group: for $a \in \gamma_{i}(G) \backslash$ $\gamma_{i+1}(G)$ define $a$ to be positive if $\gamma_{i+1}(G)+a$ is positive in $\gamma_{i}(G) / \gamma_{i+1}(G)$. Then $\gamma_{n}(G)$ is a convex central 1-subgroup.

\section{Permutational products of 0-groups}

To form the permutational product of totally ordered groups with certain normal convex subgroups amalgamated we need not resort to the Holland representation of an 1-group. We may proceed directly, as for groups.

Let $\left\{G_{i}: i \in I\right\}$ be a set of 0 -groups, $\left\{H_{i}: i \in I\right\}$ a set of normal convex subgroups, $H$ an 0-group and, for each $i$, let $\theta_{i}$ be an 0 -isomorphism of $H$ onto $H_{i}$. For each $i$, select a set of representatives $C\left(H_{i}\right)=\left\{h_{i}, \alpha\right\}$ of the right cosets of $H_{i}$ in $G_{i}$. Let $T$ be the cartesian product of $H$ with the $C\left(H_{i}\right)$, considered as the set of chcice functions $p$ from $I \cup\{\alpha\}$ such that $p(\alpha) \in H, p(i) \in C\left(H_{i}\right)$. We order $T$ by first well ordering $I$ and then defining $p>q$ if $p(i)>q(i)$, where $i$ is the first element of $I$ for which $p(i) \neq q(i)$, or $p(i)=q(i)$ for all $\mathrm{i} \in I$ and $p(\alpha)>q(\alpha)$.

For any element $g_{i}$ of $G_{i}$ we define an element $\rho_{g_{i}}$ of $P(T)$ as follows: $p \rho_{g_{i}}=q$ where $p(i)+(p(\alpha)) \theta_{i}+g_{i}=q(i)+(q(\alpha)) \theta_{i}$, and $q(j)=p(j)$, for $i \neq j \in I$.

Now define $\rho_{i}: G_{i} \rightarrow P(T)$ by $g_{i} \rho_{i}=\rho_{g_{i}}$. Then, for each $i, \rho_{i}$ is an 0-isomorphism of $G_{i}$ into $P(T)$ such that $\theta_{i} \rho_{i}=\theta_{j} \rho_{j}$, for all $i, j$ and $G_{i} \rho_{i} \cap G_{j} \rho_{j}=$ $\bigcap_{k} G_{k} \rho_{k}=H \theta_{i} \rho_{i}$.

We call the 1-subgroup $P_{0}$ of $P(T)$ generated by the set of $G_{i} \theta_{i}$ the 1-permutational product of the 0-groups $G_{i}$ with amalgamated subgroups $H_{i}$. In general, $P_{0}$ is not an 0-group. However,

THEOREM 3.1. If, for each $i, H_{i}$ is in the centre of $G_{i}$ then $P_{0}$ is an 0-subgroup of $P(T)$. If each $G_{i}$ is abelian so is $P_{0}$.

Proof. It is straightforward to show that if $H_{i}$ is in the centre of $G_{i}$, for all $i$, then elements from distinct $G_{i} \rho_{i}$ commute. Hence, if the $G_{i}$ are themselves abelian, so will the 1-subgroup generated by the $G_{i} \rho_{i}$ be abelian.

It then follows that the group generated by the $G_{i} \rho_{i}$ is just the generalized direct product $D$ of the $G_{i} \rho_{i}$ and the order induced on $D$ can be defined directly as follows: Let $\rho=\rho_{g_{1}} \rho_{g_{2}} \cdots \rho_{g_{n}} \in D$, where $g_{\alpha} \in G_{i_{\alpha}}$; we may assume that $i_{1}<i_{2}<\cdots<i_{n}$ in the order on $I$; then $\rho>1$ if and only if cither $g_{\alpha}>0$, where $i_{\alpha}$ is the first index such that $g_{\alpha} \notin H_{i_{\alpha}}$ or $g_{\alpha} \in H_{i_{\alpha}}$, for $i_{\alpha}$, and $g_{1} \theta_{i_{1}}^{-1}+g_{2} \theta_{i_{2}}^{-1}+$ $\cdots+g_{n} \theta_{i_{n}}^{-1}>0$ in $H$. This is easily seen to endow $D$ with a total order. Hence, $P_{0}$ is just the subgroup generated by the $G_{i} \rho_{i}$ and is totally ordered.

We give the following example to illustrate that even if the $H_{i}$ are normal abelian convex subgroups of the 0 -groups $G_{i}$, then the 1 -permutational product 
$P_{0}$ of the $G_{i}$ with the $H_{i}$ amalgamated need not be an 0-group. It suffices to produce an element not comparable to 1 .

Example. Let $Z^{R}$ denote the group of functions $f: R \rightarrow Z$ of finite support (where $R=$ real numbers, $Z=$ additive group of integers), ordered by: $f>0$ if $f(a)>0$ where $a$ is the largest number in the support of $f$. Let $\alpha, \beta \in P(R)^{+}$be such that $[\alpha, \beta]=\alpha^{-1} \beta^{-1} \alpha \beta$ is not comparable to 1 ; that is, such that for some $a, b \in R$

$$
a[\alpha, \beta]>a \text { and } b[\alpha, \beta]<b .
$$

The graphs of such a pair are given in Figure 1.

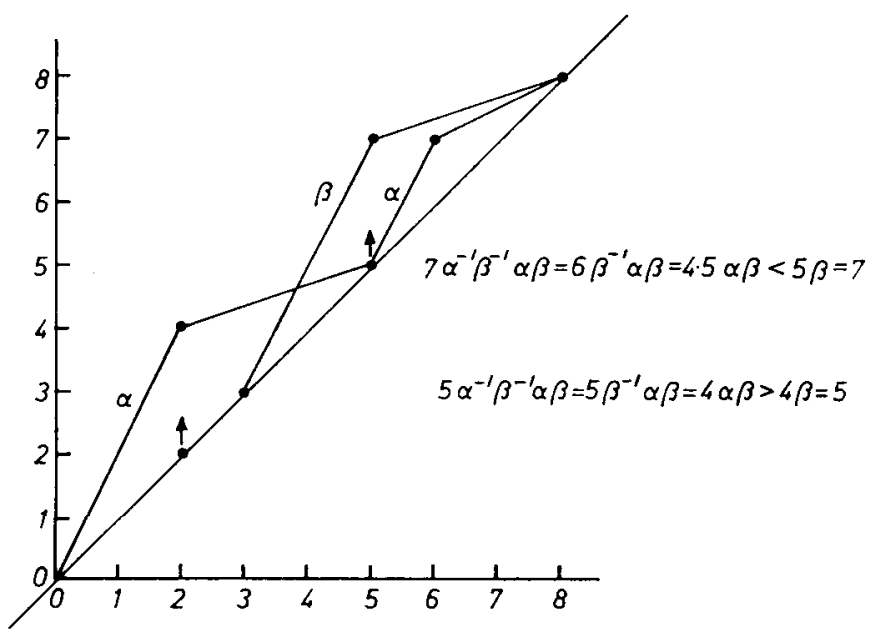

Figure 1

These induce automorphisms $f \rightarrow f^{x}, f \rightarrow f^{\beta}$ of $Z^{R}$ where

$$
f^{\alpha}(x)=f\left(x \alpha^{-1}\right), f^{\beta}(x)=f\left(x \beta^{-1}\right) \text {. }
$$

Let $G_{1}=Z^{R} \times{ }_{\varphi} Z$, the semidirect product of $Z^{R}$ with $Z$ via the isomorphism $\varphi: Z \rightarrow$ Aut $Z^{R}$ where $1 \varphi=\alpha$. Similarly, let $G_{2}=Z^{R} \times{ }_{0} Z$ where $1 \theta=\beta$. Then $G_{1}$ and $G_{2}$ are subgroups of the wreath product $G=(Z, Z) w r(R, P(R))$ of the permutation groups $(Z, Z),(R, P(R))$ where $Z$ acts on $Z$ by translation and $P(R)$ acts on $R$ naturally.

Now form the 1-permutational product $P_{0}$ of $G_{1}$ and $G_{2}$ amalgamating $Z^{R}$, with $\rho_{1}: G_{1} \rightarrow P_{0}$ and $\rho_{2}: G_{2} \rightarrow P_{0}$. We take the obvious representatives of the cosets of $Z^{R}$ and order $I=\{1,2\}$ in the natural manner. Let $f_{a}, f_{b} \in Z^{R}$, be such that

$$
f_{a}(x)\left\{\begin{array} { l } 
{ 1 \text { if } x = a , } \\
{ 0 \text { otherwise } }
\end{array} \quad f _ { b } ( x ) \left\{\begin{array}{l}
1 \text { if } x=b \\
0 \text { otherwise }
\end{array}\right.\right.
$$


Then $f_{a}^{[\alpha, \beta]} \in Z^{R} \subseteq G$ is such that

$$
f_{a}^{[\alpha, \beta]}(x)=f_{a}\left(x[\alpha, \beta]^{-1}\right)= \begin{cases}1 & \text { if } x=a[\alpha, \beta] \\ 0 & \text { otherwise }\end{cases}
$$

and so $f_{a}^{[\alpha, \beta]}>f_{a}$. Similarly $f_{b}^{[\alpha, \beta]}<f_{b}$.

Now consider the element $\left[x \rho_{1}, y \rho_{2}\right]$ of $P_{0}$, where $x=(1,0) \in G_{1}$ and $y=(1,0) \in G_{2}$. Then, for the element $\left(f_{a}, 0,0\right)$ of $T$, where $T$ is defined as in Theorem 3.1,

$$
\begin{aligned}
\left(f_{a}, 0,0\right)\left[x \rho_{1}, y \rho_{2}\right] \\
\quad=\left(f_{a}, 0,0\right)\left(x \rho_{1}\right)^{-1}\left(y \rho_{2}\right)^{-1}\left(x \rho_{1}\right)\left(y \rho_{1}\right) \\
=\left(f_{a} \alpha^{-1},(1,0), 0\right)\left(y \rho_{2}\right)^{-1}\left(x \rho_{1}\right)\left(y \rho_{1}\right) \\
\quad=\cdots \\
\quad=\left(f_{a}^{[\alpha, \beta]}, 0,0\right)>\left(f_{a}, 0,0\right) .
\end{aligned}
$$

On the other hand $\left(f_{b}, 0,0\right)\left[x \rho_{1}, y \rho_{2}\right]=\left(f_{b}^{[\alpha, \beta]}, 0,0\right)>\left(f_{b}, 0,0\right)$. Thus the element $\left[x \rho_{1}, y \rho_{2}\right]$ in $P_{0}$ is not comparable to 1 and $P_{0}$ is not an 0 -group.

Note: In the case of groups, the permutational product of two groups $A$ and $B$ amalgamating a subgroup $H$, central in each, is just the central product of the two groups. However, there is some difficulty in defining the central product if $A$ and $B$ are 1-groups. If $H$ is in the centres of $A$ and $B$ then the central product of $A$ and $B$ amalgamating $H$ is $C=A \times B / N$ where $N$ is the subgroup of $A \times B$ generated by $\{(h,-h): h \in H\}$. However, if we wish to endow $C$, or some similar group, with a lattice order by first endowing $A \times B$ with the cardinal order and then factoring, then the kernel $\bar{N}$ has to be an 1-ideal and contain $N$. Thus if $h>0$ we must have $(h, 0)=(h,-h) \vee 0$ and $(0,-h)=(h,-h) \wedge 0$ in $\bar{N}$ and so the 'embeddings' of $A$ and $B$ in $A \times B / \bar{N}$ would not be monomorphisms.

\section{Amalgams of representable 1-groups}

As remarked in Section 2, Conrad has shown that if $H$ is a convex 1-subgrcup of an 1-group $G$ then the mapping $\sigma: M \rightarrow M \cap H$ is a bijection of the set of prime subgroups of $G$ not containing $H$ onto the set of prime subgroups of $H$. Clearly, if $M$ is an 1-ideal of $G$ then $M \sigma$ is an 1-ideal of $H$, although the converse need not be true. For want of a more appropriate name, if $\sigma^{-1}$ maps 1-ideals of $H$ onto 1-ideals of $G$ then we shall call $H$ an l.e. convex 1-subgroup of $G$ (short for: 1-ideals are extendable from $H$ to $G$ ). For instance, if all the prime subgroups of $G$ are normal then any convex 1-subgroup of $G$ would be an 1.e. convex 1subgroup of $G$.

An 1-group $G$ is called representable if there exists an 1-isomorphism of $G$ onto a subdirect sum of a cardinal sum of 0-groups. Conrad [2] has shown that $G$ is representable if and only if every regular 1-ideal is a primc subgroup. A regular 
1-ideal of an 1-group is an 1-ideal which is maximal in the lattice of 1-ideais with respect to not containing some element. If $H$ is an 1.e. convex 1-subgroup of a representable $G$ then it is not difficult to show that $\sigma$ is also a bijection of regular 1-ideals of $G$ not containing $H$ onto the regular 1-ideals of $H$.

THEOREM 4.1. Let $\left[G_{i} ; H ; \theta_{i} ; H_{i}\right]$ be an amalgam of 1 -groups where each $G_{i}$ is representable and each $H_{i}$ is a normal l.e. convex 1-subgroup of $G_{i}$. Then the amalgam is embeddable in a 1-group.

Proof. For each $i$, let $\sigma_{i}$ be the bijection, described above, of the prime subgroups of $G_{i}$ not containing $H_{i}$ onto the prime subgroups of $H_{i}$.

Let $\left\{L_{\gamma}: \gamma \in \Gamma\right\}$ be the set of regular 1-ideals of $H$; then $\left\{L_{\gamma} \theta_{i}: \gamma \in \Gamma\right\}$ is the set of 1egular 1-ideals of $H_{i}$, and $\left\{L_{\gamma} \theta_{i} \sigma_{i}^{-1}: \gamma \in \Gamma\right\}$ is the set of regular 1-ideals of $G_{i}$ not containing $H_{i}$; let $\left\{L_{i, \delta}: \delta \in \Gamma_{i, 1}\right\}$ be the set of regular 1-ideals of $G_{i}$ con-. taining $H_{i}$.

We denote by $\varphi_{i}$ the natural 1 -isomorphism of $G_{i}$ into

$$
\bar{G}_{i}=\left(\sum_{\gamma \in \Gamma} \boxplus G_{i} / L_{\gamma} \theta_{i} \sigma_{i}^{-1}\right) \boxplus\left(\sum_{\delta \in \Gamma_{i, 1}} \boxplus G_{i} / L_{i, \delta}\right),
$$

where, for 1-groups $M_{\alpha}, \sum_{\alpha \in A} \boxplus M_{\alpha}$ denotes the (unrestricted) cardinal sum of the 1 -groups $M_{\alpha}$. We denote by $\pi_{\gamma}, \pi_{i, \delta}$ the projections of $\bar{G}_{i}$ onto $G_{i} / L_{\gamma} \theta_{i} \sigma_{i}^{-1}$ and $G_{i} / L_{i, \delta}$, respectively.

Now, for each $\gamma \in \Gamma$, we have the amalgam of 0 -groups $\left[G_{i} / L_{\gamma} \theta_{i} \sigma_{i}^{-1} ; H / L_{\gamma}\right.$; $\left.\theta_{i} \pi_{\gamma} ; H_{i} / L_{\gamma} \theta_{i}\right]$ where, by $\theta_{i} \pi_{\gamma}$, we mean the naturally induced 1 -isomorphism of $H / L_{\gamma}$ onto $H_{i} / L_{\gamma} \theta_{i}$. By Section 3, we can always embed such an amalgam in a 1-group. So, for each $\gamma$, choose such an embedding $\left\{\rho_{i, \gamma}\right\}$ into an 1-group $K_{\gamma}$.

We write

$$
K=\left(\sum_{\gamma \in \Gamma} \boxplus K_{\gamma}\right)+\sum_{i \in I} \boxplus\left(\sum_{\delta \in \Gamma_{i, 1}} \boxplus G_{i} / L_{i, \delta}\right) .
$$

We denote by $l_{\gamma}, l_{i, \delta}$ the injection of $K_{\gamma}$ and $G_{i} / L_{i, \delta}$ into $K$. Then, for each $i$,

$$
\Psi_{i}=\left(\prod_{\gamma \in \Gamma} \varphi_{i} \pi_{\gamma} \rho_{i, \gamma} l_{\gamma}\right) \times\left(\prod_{\delta \in \Gamma_{i, 1}} \varphi_{i} \pi_{i, \delta} l_{i, \delta}\right)
$$

is an 1-iscmorphism of $G_{i}$ into $K$ and $\left\{\Psi_{i}\right\}$ is an embedding of $\left[G_{i} ; H ; \theta_{i} ; H_{i}\right]$ into $K$.

\section{References}

[1] G. Birkhoff, Lattice theory (rev. ed. 1967, Amer. Math. Soc. Colloquium Pub. 25).

[2] P. Conrad, 'Archimedean extensions of lattice ordered groups', J. Indian Math. Soc. (N.S.) 30 (1966), 131-160 (1967).

[3] P. Conrad, Introduction à la théorie des groupes réticulés (Lecture Notes, Université de Paris).

[4] L. Fuchs, Partially ordered algebraic systems (Pergamon Press (1963)).

[5] G. Higman, B. H. Neumann and Hanna Neumann, 'Embedding theorems for groups', J. London Math. Soc. 24 (1949), 247-254. 
[6] C. Holland, 'The lattice ordered group of automorphisms of an ordered set', Michigan Math. Jour. 10 (1963), 399-408.

[7] J. M. Howie, 'Embedding theorems with amalgamations for semigroups', Proc. Lond. Math. Soc. (3) (1962), 511-534.

[8] T. Lloyd, Lattice ordered groups and 0-permutation groups, Thesis, Tulane University, 1964.

[9] B. H. Neumann, 'An essay on free products of groups with amalgamations', Phil. Trans. Roy. Soc. 246 (1954), 503-554.

[10] R. S. Pierce, Introduction to the theory of abstract algebras (Holt, Rinehart and Winston (1968)).

Department of Mathematics

Simon Fraser University

British Columbia, Canada 